\title{
Relational and Substantival Ontologies, and the Nature and the Role of Primitives in Ontological Theories
}

\author{
Jiri Benovsky
}

Received: 20 March 2009/Accepted: 2 February 2010/Published online: 5 March 2010

(C) Springer Science+Business Media B.V. 2010

\begin{abstract}
Several metaphysical debates have typically been modeled as oppositions between a relationist approach and a substantivalist approach. Such debates include the Bundle Theory and the Substratum Theory about ordinary material objects, the Bundle (Humean) Theory and the Substance (Cartesian) Theory of the Self, and Relationism and Substantivalism about time. In all three debates, the substantivalist side typically insists that in order to provide a good treatment of the subject-matter of the theory (time, Self, material objects), it is necessary to postulate the existence of a certain kind of substance, while the other side, the relationist one, characteristically feels that this is an unnecessary expense and that one can get the job done in an ontologically cheaper way just with inter-related properties or events. In this paper I shall defend the view that there is much less of a disagreement between relational ontologies and substantival ontologies than it is usually thought. I believe that, when carefully examined, the two sides of the debate are not that different from each other, in all three cases of pairs of views mentioned above. As we will see, both the relational side and the substantival side work in the same way, suffer from and answer the same objections, and are structurally extremely similar. It will be an important question — one that I shall discuss in detail, and that is indeed the main point of interest for me in this paper-whether this means that the two sides of the debate are somehow 'equivalent' or not, and what 'equivalent' could mean.
\end{abstract}

J. Benovsky $(\bowtie)$

Department of Philosophy, University of Fribourg, Office 5242A,

Miséricorde, Fribourg, Switzerland

e-mail: jiri@benovsky.com

URL: http://www.unifr.ch/philo/modern-contemporary/benovsky/; www.jiribenovsky.org 


\section{Relationist and Substantival Theories}

Throughout the history of occidental philosophy, several debates have been modeled as oppositions between a relationist approach and a substantivalist approach. Such debates include:

- Relationism vs. Substantivalism about time

- The Bundle Theory vs. the Substratum Theory about ordinary material objects

- The Bundle (Humean) Theory vs. the Substance (Cartesian) Theory of the Self ${ }^{1}$

In all three debates, the substantivalist side typically insists that in order to provide a good treatment of the subject-matter of the theory (time, Self, material objects), it is necessary to postulate the existence of a certain kind of substance ${ }^{2}$ that is required to account for some important issues such as particularity, individuation, unity, independence, persistence, ... and that allows thus to solve some puzzle cases, intriguing phenomena, or philosophical problems (of which I shall discuss some examples below). Without an underlying substance, the friends of the Substratum Theory feel that properties would go 'floating free' and objects like tables would lack particularity, the Self would lack unity, and nothing could genuinely persist through time while undergoing intrinsic change. Substantivalists about time would analogously feel that events and things need to 'occur at' or 'be located at' times that need to be substantial enough to be able to 'support' them or 'contain' them.

The other side, the relationist one, typically feels that this is an unnecessary expense and that one can get the job done in an ontologically cheaper way. Relationists will thus deny the need for any substance, they will claim that it is a mysterious thing that we should spare ourselves, and that it is enough to use the entities that substantivalists take to be 'had' or 'united' by the substance (properties, events, ...) and explain how these are inter-related to account for all phenomena, puzzle cases, and philosophical problems. According to this side, that often declares itself to be more respectful of Occam's Razor, it is enough to have properties tied together by a special relation of 'compresence' to get ordinary objects or Selves (that are bundles of properties) and it is enough to have events related by a special relation of simultaneity to get times (simultaneity classes of events).

Not only are the three debates similar in nature, but we also step on very similar arguments and objections in all three cases. To mention now only one, a large literature has been devoted to the discussion of an alleged great difficulty for bundle theories of material objects: the problem with Identity of Indiscernibles, which parallels the problem often raised against the relationist theory of time: the case of 'Time without Change'. Both problems yield the same difficulty, except that in one

\footnotetext{
${ }^{1}$ Although I talk about "the Cartesian view" and "the Humean view", I will not really be concerned with Cartesian or Humean exegesis. I think that what I will say about these views is (at least) very close to views Descartes and Hume actually held, but exact interpretation of their writings is a tricky endeavour that I shall not attempt.

${ }^{2}$ The word "substance" is a tricky one, and it often means very different things in the mouths of different philosophers. I shall use it as a synonym of "substratum", and when it matters (see below) I will say precisely what it refers to.
} 
case the question is about how to account for numerical diversity of objects that have the same properties (that are qualitative duplicates) and in the other case the question is about how to account for numerical diversity of times (instants) that 'have' the same events, that is, at which the very same events occur during a period of time without change. (I will discuss this in detail in Sect. 2.)

In this paper I shall defend the view that there is much less of a disagreement between relational ontologies and substantival ontologies than it is usually thought. I believe that, when carefully examined, the two sides of the debate are not that different from each other, in all three cases of pairs of views mentioned above. As we will see, both the relational side and the substantival side work in the same way, suffer from and answer the same objections, and are structurally extremely similar. It will be an important question - one that I shall discuss in detail, and that is indeed the main point of interest for me in this paper-whether this means that the two sides of the debate are somehow 'equivalent' or not, and what 'equivalent' could mean. Principally, I will ask what grounds we can have either to say that the two sides are metaphysically equivalent and mere terminological variants of each other or to say that they are metaphysically different views but that they are so similar that it is epistemically under-determined which one we should choose. In the context of the recent debate in meta-metaphysics, this choice can be seen as one between two possible answers that have been given to the question of whether metaphysical debates are really substantive or whether they are merely verbal. This question has received various answers, giving rise to two 'extreme' and two 'moderate' views. On the two opposite sides of the spectrum of the debate lie the two 'extreme' views: first, the realist view recently defended by Sider (2001, 2007, 2008) defends the claim that metaphysical disputes are substantive and that metaphysical questions have objective answers, while the sceptical anti-realist view defended in the recent debate in different ways by Chalmers (2008) and Yablo (2008) claims that metaphysical questions do not have objective answers, they can be formulated and answered in different frameworks and there is no fact of the matter as to which framework is correct-thus, metaphysical claims lack truth-value. In between these two 'extreme' views lie two 'moderate' ones. Bennett (2008) defends an epistemicist view that claims that at least some metaphysical questions have genuine objective answers but that often we cannot discover them and that consequently there is often little reason or no reason at all to go for one side rather than the other, and Hirsch $(2005,2007,2008)$ defends a moderate anti-realist view that claims that many metaphysical debates are merely verbal disputes where the disputants seem to claim different things but in fact they are making the same claims only formulated in different ways, or different languages. It is these two latter views that I will be most interested in.

The strategy of this paper is the following. For each one of the three debates, I shall take one central point of traditional strong disagreement between the two sides, and I will show that, far from being a real divide, the point actually shows us how similar the two sides are. In Benovsky (2008, and forthcoming), I was able to examine more such points separately for these debates, but given constraints on the length of this paper I will have to restrict myself here to one central point per debate only. This paper in itself is thus not complete enough to draw any 'equivalence' 
conclusion between the two sides of a debate, since this would require going through all of the points of alleged disagreement between them; rather the focus of this paper is to make plausible and illustrate this 'equivalence' claim, and discuss in detail what drawing such a conclusion amounts to, what it means, what it depends upon, and what its consequences are. One central point of discussion that, as we will see, plays a vital role with respect to this meta-metaphysical question will be the nature and the role of primitives in metaphysical theories. Primitives play a crucial role in any theory and it is by examining one's theory primitives and the way they work that important steps towards an 'equivalence' claim can be made.

Let us start with Relationism and Substantivalism about time.

\section{Relationism and Substantivalism About Time}

According to the substantivalist theory of time, events and things 'are located at' or 'occur at' different instants of time and these instants are seen as being independent of the events or things they 'contain'. Time is then a substance composed of such instants. It is thus easy for this view to account for periods of time without change, that is, for periods where all change stops and the universe is 'frozen' during a certain interval of time, since instants are not dependent on the changes that occur at them. Similarly, the view is also typically taken to be able to accommodate periods of 'empty time', that is, periods where no events occur at all. Metaphorically, time is here conceived of as a container that can contain events and things, but that is capable of not containing anything.

In opposition to substantivalism, the relationist theory of time denies the (need for the) existence of such a substance, and claims that all there is are events and things. Instants are not 'containers', rather they are constructed out of events-an instant is a collection of simultaneous events (a simultaneity class of events). Thus, it is typically claimed that relationism cannot accommodate the possibility of empty time, since the very existence of instants depends on the existence of something 'occurring at' them, nor the possibility of time without change, which is the point I shall now start with.

Let us imagine a Shoemaker-like universe ${ }^{3}$ where its inhabitants have very good reasons to believe that every 30 years all change comes to a stop for $1 \mathrm{~h}$. If you are not convinced by Shoemakers' argument, just think of it as a brute metaphysical possibility: in this possible world, a 'global freeze' happens every 30 years for $1 \mathrm{~h}$, and perhaps its inhabitants never get to realize it. One can also think of this scenario as just a useful thought experiment that helps us to articulate better the debate between relationists and substantivalists. Typically, it has been argued that relationism cannot accommodate such a possibility (and those who find this possibility genuinely plausible consequently take it to be an argument against this view). At least prima facie, this seems like the right thing to say: if there are no changes, if the universe is 'frozen', it appears that under relationism there is no way

\footnotetext{
3 See Shoemaker (1969).
} 
of having different instants that would constitute a $1 \mathrm{~h}$ long series, since these instants would all turn out to be one and the very same instant.

In what follows I intend to show that far from not being able to accommodate the possibility of global freezes, relationism can actually accommodate it in the same way substantivalism does. But my point here is not to defend relationism, rather I am interested in defending the view that the two sides of this debate about the nature of time are extremely similar to one each other. My strategy is thus very different from, for instance, Forbes' (1993) strategy that 'modalizes' relationism, that is, includes other-worldly events into the making of this-worldly instants, in order to be able to distinguish instants modally during periods of global freezes. Such a strategy is one that wants to defend relationism by repairing it and improving it, whereas I want to claim that relationism as it stands can face the case of time without change in exactly the same way substantivalism does.

Let us first ask: how exactly does substantivalism accommodate the possibility of global freezes? If there is a global freeze and all change stops, how can time continue to flow? What we need here is to be able to accommodate the possibility of a series of non-identical instants, instead of just one instant. But how can instants that compose a series during a global freeze be distinguished? They cannot be distinguished by the events/changes that occur at them, since there are no changes and the events are all the same at all instants during this series. Qualitatively speaking, all of them are indiscernible, and so if qualitative identity were the relevant criterion for distinguishing instants, there would only be one instant instead of a $1 \mathrm{~h}$ long series. So what makes different instants different is something else than qualitative differences: instants are primitively numerically distinct entities that do not require to be distinguished qualitatively (since, in the first place, they do not have a qualitative nature such that they could be distinguished in a qualitative way). Metaphorically speaking, instants are containers that are in themselves qualitatively indistinguishable and that, during a global freeze, contain qualitatively indistinguishable stuff, but that are primitively numerically distinct.

Instants conceived of in such a way are what I will call "problem-solvers". In short, a problem-solver is a primitive of a theory that allows us to solve a problem. In general, it is probably the case that all primitives are, at least to some extent, problem-solvers, for primitives are typically introduced in any theory to do an explanatory job that they manage to do by having the primitive capacity to do so. In the case of substantivalism, how can the theory account for the possibility of periods of time without change? By using its primitive notion of instants that are qualitatively indistinguishable but that are primitively numerically distinct. This premise is thus a "problem-solver" since without appealing to it the theory would not be able to accommodate the possibility of global freezes and since it succeeds to achieve its end only in virtue of the postulation that it can do so. It may seem from what I just said that I take substantivalism to be an unappealing view that does not know better than primitively postulating solutions to its theoretical challenges. But this is not so: every theory has its primitives (we will see more examples below), and every theory has the right to do so, since without the use of such problemsolvers we would not be able to get very far in the construction of metaphysical theories (the examples below will also make this clear). 
Now, what about relationism? Since under relationism instants are simultaneity classes of events, that is, they are bundles ${ }^{4}$ of events tied together by a relation of simultaneity, they are individuated by these events and the relation-consequently, instants have a qualitative nature and they can be distinguished by the events they contain. But not in the case of a global freeze: when such a period of time without change occurs, all instants that form the $1 \mathrm{~h}$ long series contain the very same events and so cannot be qualitatively distinguished, and so where we wanted to have a series of distinct instants we turn out to have only one instant-this is why the objector argues that relationism is unable to account for the possibility of time without change.

But let us look more closely at how relationism works; especially, let us examine what the relation of simultaneity is and what it does. The theoretical role of this relation is the functional role to take events as input and provide instants as output. Each instant has its own such relation, it cannot be one and the very same relation for all instants, since otherwise, global freeze or not, there would ever only be one 'big' instant containing all (past, present, future) events that would be simultaneous with one each other (alternatively this job can be done by different instances of the same relation ${ }^{5}$ ). This is then a schema of the structure of relationism:

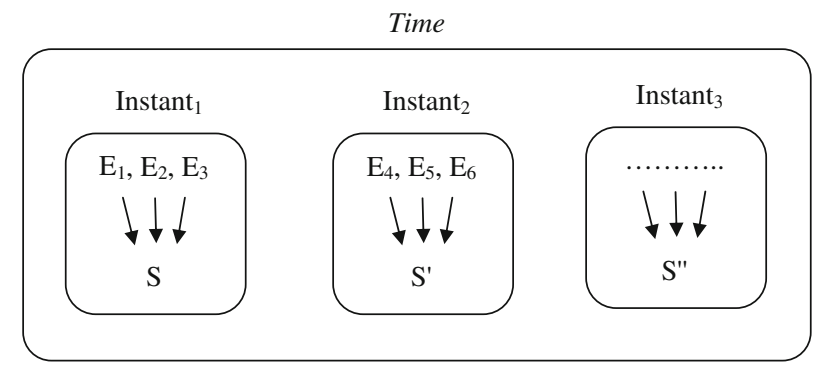

\footnotetext{
${ }^{4}$ Not sets, since instants are not abstract entities according to this view.

5 If properties and relations are tropes, the situation is obvious: tropes being non-repeatable entities it would not even be possible for the relation of simultaneity to be one and the same for different instants, so what we have here are exactly resembling and numerically distinct tropes of simultaneity, one per instant. If properties and relations are universals, there are two possibilities: either the relation of simultaneity is one and the very same relation for all instants, or it is a different universal for each instant. As suggested, the former possibility yields difficulties even if no global freeze occurs - the case of a global freeze is just the most salient case where these difficulties become the most apparent-so it is the latter that should be endorsed anyway. Alternatively, claiming that there are numerically different instances of one universal of simultaneity could also do the job. Such moves are similar to a strategy that Paul (forthcoming) explores with respect to the Bundle Theory of objects, when she says: "[...] properties are shared, while property instances are primitively individuated. On this approach the explanation of the possibility of the qualitative indiscernibility of the spheres in $\mathrm{W}$ is based on an underlying identity of properties, while the numerical difference between the spheres reductively supervenes upon the numerical difference of the property instances in each bundle". What is important for me here is that there is always something numerically different for each instant that is responsible for tying up together the events to make up the instant.
} 
Time is a series of instants that are made out of events tied together by a relation of simultaneity, a different one for all instants. Now, when a global freeze occurs, there are no changes, and so the events that compose the instants are the same, but there is nothing there that prevents relationism from accommodating the claim that there is a $1 \mathrm{~h}$ series of numerically distinct instants: granted, this series is formed by instants that cannot be distinguished by the events they contain, but they can be distinguished by the relation of simultaneity that is a different one in two different instants (even when no freeze is going on). The relation of simultaneity individuates instants as well as events do, and instants can thus be numerically distinguished because they contain numerically distinct relations of simultaneity, even if they cannot be qualitatively distinguished (that is, distinguished by appeal to the events the contain). One may ask: but how is one relation of simultaneity distinguished from another relation of simultaneity? (I mean how, not why, we have seen why above.) How is it not the case that since presumably one relation of simultaneity is qualitatively indistinguishable from another, it just turns out, contrary to what we would want here, to be one and the very same relation for all instants? Exactly as in the case of substantivalism, the answer is: the different relations of simultaneity can be said to be primitively numerically distinct. So, as before, relationism contains here a problem-solver that parallels the one that is appealed to by substantivalists: it is only by appealing to their primitive problem-solving machinery that distinguishes primitively numerically different instants that both relationism and substantivalism can accommodate the theoretical challenge of the possibility of time without change. Both problem-solvers have the same function with respect to the problem concerning time without change, and consequently they are theoretically equivalent. The fact that one side of the debate calls its problem-solver a "substance" or a "container" or a "time", while the other side calls its problem-solver a "relation of simultaneity", does not change the fact that when one looks closely at what these primitive problem-solvers are doing in the theory, they do turn out to be (theoretically, functionally) indistinguishable: they both have the function to make different instants different. But before I go any further and before I discuss in detail what the consequences of this are (in Sect. 4), let us turn our attention to an analogous debate that exhibits a relational and a substantival side, namely, the Bundle Theory and the Substratum Theory about material objects and about the Self.

\section{The Bundle Theory and the Substratum Theory}

While the substratum theory postulates two kinds of components to make up material objects like, say, a table or a person, the bundle theory postulates only one. Sometimes the substratum theory is also called the "substance-attribute view" to make this apparent: there is a substance (or "substratum", or "underlying subject", or "bare particular") and there are properties (attributes) that are had by this substance. (I shall use "substratum" rather than "substance", the latter being a tricky word in philosophy meaning very different things in the mouths of different philosophers.) Traditionally opposed to this, the bundle theory wants itself to be 
more economical and uses one kind of component only: the same properties that the friends of substrata take to be had by the substratum, but 'only' tied together by a special $n$-adic property often called "compresence". Reality is thus constituted fundamentally only by properties, and objects like tables or persons are 'just' bundles of those.

I take it that the overall structural similarities between substantivalism and the substratum theory, and between relationism and the bundle theory, are quite obvious. The former two theories do their job by having some sort of primitive substance that somehow supports what is in need of being supported (events in one case, properties in the other); the latter two views both reject this conception and claim that events or properties need not be supported or had by anything and that the notion of a primitive supporting substance can be very well eliminated and replaced by collections of these 'unsupported' entities (that is, by a primitive relation of 'compresence' or 'simultaneity').

Depending on how one conceives of the compresence relation (the 'bundling' relation) and of the nature of properties, the bundle theory comes in different versions. Most relevantly, both the bundle theory and the substratum theory come in a version where properties are tropes (and in this case the compresence relation has to be a numerically distinct relation in every particular object, since a trope is a nonrepeatable entity) and in a version where properties are universals (and in this case there are both the possibilities that the relation of compresence is a different one for every object, or that it is one and the same variably polyadic relation for all objects). In what follows, I shall remain neutral with respect to any preference of tropes or universals.

The bundling relation used by the bundle theory goes around under different names like "compresence", "consubstantiation", "co-instantiation", "togetherness", "collocation", "co-personality" (in the special case of the Humean bundle theory of the Self)—but all these different names do not hide different conceptions or different analysis or what this bundling relation is; rather, most typically, all bundle theorists take it as being a primitive. Thus, analogously to what we have seen above in the case of relationism and substantivalism, what is important is not so much (or even not at all, as we shall see below) how this relation is called or what it is, but rather what it does: it is a unifying device, that is, a primitive bit of theory that has the function within the theory to take properties to make up objects. Putting it as an objection to the bundle theory, Peter Simons (1994, p. 371) expresses this quite clearly when he says that "[...] all we are saying is that a bundle of tropes is held together by whatever relation holds it together. This is really giving up." Forget now about the "giving up" part of Simons' claim, and concentrate on the nature of the bundling relation: it is, to paraphrase Locke, a "we-know-not-what" but ... it is a "we-do-know-what-it-does" ! We know its theoretical role: what it does is that it ties together properties to make up objects, and that's all we need to be told, the bundling relation being a primitive device introduced by the bundle theorist precisely for this purpose.

In the case of the substratum theory, things are exactly the same way, and it is even more obvious: the substratum also goes around under different names like "naked particular", "bare particular", "thin particular", or "substance" but we are 
typically not told anything in place of an analysis or explanation of the nature of the substratum, it is also a primitive whose theoretical role is to support properties of an object. Its theoretical function is, as in the case of the bundling relation, to take properties to make up objects - that's what it does, that's its main job. As before, it is a "we-know-not-what" in the sense that it is ontologically primitive and unanalyzable, but it is a "we-do-know-what-it-does". As in the case of the bundling relation, the substratum is thus individuated by its theoretical role: it is a unifying device. Both the bundle theory and the substratum theory thus have a unifying device, a primitive and under-defined one, an entity whose purpose is to tie or glue together properties of a single object.

With respect to the issue about particularity and unity of objects, both the bundle theory and the substratum theory thus contain a problem-solver in the same sense in which relationism and substantivalism contain one. Another way to see this is to ask how the theory accounts for numerical diversity of different substrata-it cannot be done qualitatively since substrata in themselves do not have any qualities, and so, here again, the question is answered by a primitivist claim ... exactly as in the case of the bundle theory's bundling relation where one can also ask what makes one such unifying device bundling together properties of an object $A$ numerically distinct from another unifying device bundling together properties of an object $B$. These primitivist strategies that appeal to problem-solvers that are now familiar to us from the discussion about relationism and substantivalism thus play a crucial role in both the substratum theory and the bundle theory with respect to the central question that both theories address, namely the question of what an object is and what makes it a particular.

One could think that the status of the bundle theory's unifying device is importantly different from the status of the substratum theory's unifying device because the former is just one among the elements of a bundle (it is a property, after all) while the latter is to be considered apart from the properties it bears-thus even if both are primitive problem-solving unifying devices, they do not play their primitive theoretical role in the same way. But, as Ehring (2001) shows, this would be misconceiving the status of the bundling relation (what follows is an almost exact quote from Ehring that I just adapted to suit my needs): The properties included in a bundle are compresent. The compresence relation, however, is not a member of the bundle like the other properties and relations because if we included compresence in the bundle, then it would itself have to be compresent with the other properties: compresence compresent with $F, G, H, \ldots$ But that either makes no sense or leads to an infinite regress. And what it shows is that compresence, exactly as the substratum, has to be considered apart from the other properties of the object, because it is a higher-order relation that has properties as relata that does not have the same theoretical role as any other properties-its status as a unifying device is different from the other properties and is the same in the bundle theory and the substratum theory.

We have now seen that the bundle theory and the substratum theory both contain a primitive problem-solver that functions in the same way with respect to the problem of unity and particularity of particulars. Let us now see that it also plays the same theoretical role in the same way with respect to another central problem: 
the persistence of ordinary objects through time and qualitative change. Elsewhere ${ }^{6}$ I have examined this in more detail, articulating both a perdurantist and endurantist versions of both views; here I will only limit myself to convey the main idea about how these problem-solvers do their job.

When an object undergoes qualitative change, that is, change in its properties, there still is some sense in which we want to be able to say that it's the same object even if it isn't qualitatively the same. Typically, it is argued that the substratum theory has no problem with such a claim - take the case of my neighbour Cyrano: he first has a big nose, then he undergoes plastic surgery, and so later on he has a small nose, but he still is one and the same object since even if his properties change (some properties are replaced by others) something always remains: the substratum. The bundle theory is traditionally unable to do anything like this: "If a thing were a set of properties, it would be incapable of change. For a thing could change its properties only if the set identical with it could change its members, but that is impossible; no set can change its members. [...] What we have is replacement of one individual by another, not change in the properties of one and the same individual." (Van Cleve 1985, p. 122-124).

But let us look more carefully at what is going on here. First, let us ask: how does the substratum theory do it to answer this objection, or more precisely how is it so that the objection allegedly does not even arise against the substratum theory? What exactly happens when Cyrano changes from being big-nosed to being small-nosed? If Cyrano is a 'thick particular', that is, a substratum + its properties, then he does not change for exactly the same reason Van Cleve claims that there is no change under the bundle theory: the thick particular with a big nose is simply replaced by something different, another thick particular with a small nose-and these two entities just are two different things, exactly as two different bundles are two different things if they do not contain the same properties. Then maybe Cyrano is the 'thin particular', that is, he only is the substratum-but in this case he cannot undergo qualitative change either since the substratum in itself does not have any qualities that could change, so no change is possible here either. So it seems that, contrary to appearances, both views suffer from the same objection for mostly the same reason: instead of genuine change we seem to have replacement (or no change at all). Now, the similarity between the two views does not stop here, since it is also the case that both views can answer this objection in a parallel way. Again, the details of this, featuring perdurantism and endurantism, I discussed elsewhere, but the core idea can be quickly put as follows: friends of the substratum theory will claim that when Cyrano undergoes qualitative change there is an object that is made up of a substratum and of properties, the properties are replaced, but something remains the same over time, the substratum, and this is what gives justice to the claim that Cyrano is one and the same object through time and through change since the substratum is the crucial element that makes him to be what he is-it is what makes him to be this particular and not some other particular. But then, if something along these lines is an acceptable account of qualitative change, the bundle theory has exactly the same strategy at hand (see how easy it is for me to write an almost

\footnotetext{
${ }^{6}$ Benovsky (2008).
} 
exact sentence here): when Cyrano undergoes qualitative change there is an object that is made up of properties tied together by a bundling relation, the properties are replaced, but something remains the same over time, the bundling relation, and this is what gives justice to the claim that Cyrano is one and the same object through time and through change since the bundling relation is the crucial element that makes him to be what he is - it is what makes him to be this particular and not some other particular. Both views can thus appeal to their 'particularizing' primitive problem-solving unifying device to face the challenge arising from cases of qualitative change, and both can do it in the same way.

Now, the meta-metaphysician raises her eyebrows: what difference does it really make to use the unifying device "substratum" or the unifying device "compresence"? This question also applies to relationism and substantivalism: what difference does it really make to use the problem-solver "relation of simultaneity" or the problem-solver "substance/instant"? Before answering this question, let us quickly examine a last illustrative case in a more precise way: the case of the bundle theory and the substratum theory of the Self with respect to the problem of fission and change over time. Indeed, until now I have shortly discussed a normal case of persistence of objects through time involving simple change in intrinsic properties. Interestingly, Shoemaker (1997) discusses a fictional case of fission (and also of teletransportation) of persons and suggests that friends of the substratum view of the Self have the natural tendency to regard these cases as not person-preserving, while friends of the bundle view of the Self typically find it natural to say that they are. If so, it could mean that there is some difference with respect to persistence through time of Selves that only becomes apparent in these extravagant cases.

But I think that there is no reason why one side should find it more natural than the other to claim that such procedures are person-preserving or not-rather, such a claim is independent of the choice between a substantival approach or a bundle-theoretic approach; it is for independent reasons that one might think (or not) that the procedure is not person-preserving and then model the situation either in terms of substrata or in terms of bundles, with no significant difference. To illustrate this, let us examine what might happen in a fictional case of fission of my neighbour Cyrano who has a big nose and who wants to undergo plastic surgery in order to have a smaller one. Unfortunately for him, just before the surgery, he undergoes fission, and since he only has money to pay the surgery once, only one of the two post-fission Cyrano(s) will be able to get a smaller nose, while the other will have to keep the big one. With respect to the identity of Cyrano, there are four possibilities: both of the resulting post-fission Selves are Cyrano(s), none of the two resulting Selves is Cyrano, or one of the two is Cyrano and the other is not. All four possibilities can be equally well modeled and accounted for by the substratum view and the bundle view; let us quickly see how this looks for the first option, the other three cases being easily done in a similar way. Since this case also involves simple change in intrinsic properties over time, it is also needed to be here more precise about which theory of persistence one is using-I shall use here the perdurantist 'worm view' that seems to me the most easily pictorially illustrative of the non-differences between the bundle theory and the substratum theory. If both postfission Selves are said to be Cyrano(s), this is how it looks under perdurantism and the bundle view: 


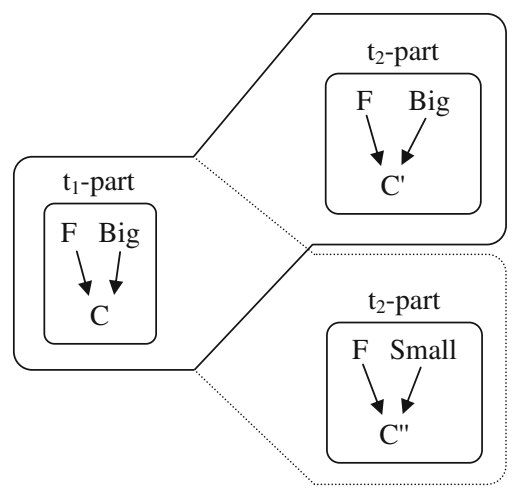

According to the perdurantist worm view, the case of fission is no more than a case of sharing an overlapping temporal part. The bundle view can easily provide such an account as pictured above where " $\mathrm{C}$ " stands for "compresence" and the substratum view can do the same-just replace the Cs with Ss ! Instead of appealing to the unifying device "compresence", one can use the unifying device "substratum" to unify Cyrano's temporal part's properties. There is no reason why one could not do this as simply as that-and the simpler this is to do, the more it points towards the idea that it just does not matter which way we put it. Under both views, it is easy to model the idea that a fission case is person-preserving and that both post-fission resulting persons are Cyrano(s), and it would be equally easy, using the same strategy, to model the other three options, under both the perdurantist and the endurantist approach. So, the question whether fission or teletransportation procedures are person-preserving must be decided on other grounds (most likely, the answer will depend on the nature of the relation that 'glues together' temporal parts of worms like Cyrano under perdurantism, and on the criteria for diachronic identity under endurantism), and then modeled in one or the other way, using with equal efficiency substrata or bundles.

The upshot of all the above considerations is, again, the following: the underlying substratum and the compresence relation (usually called the "co-personality relation" in the case of Selves) play the same theoretical role. Thus, because both the relationist side and the substantivalist side use their unifying device in the same way, they have exactly the same means to face the case of intrinsic change over time and the case of fission in a parallel way.

All the cases we have seen are illustrative of a phenomenon that affects relational and substantival ontologies in general: objections traditionally raised against one side actually have a sneaky tendency to arise for the other side as well, and can be answered (or cannot be answered) in the same way by both parties. Relevantly, in all such cases, the job is done by appeal to the theory's primitives. But if these are theoretical entities individuated by their theoretical role, and if this role is the same, then it becomes really hard to see what difference there is between the two sides of the debate. This claim rests upon the idea that primitives are individuated by their functional role rather than by their nature, which is an important point to which I shall turn now. 


\section{On Primitives and Equivalences}

The view that underlies my way of seeing all the illustrative cases above is a view about the nature of primitives in metaphysics such as the problem-solvers we have seen that takes very seriously the functional role they play in the theory. By its very nature, a primitive being primitive, it is non-analysable and we are not really given any information concerning its nature; we are told what it does rather than what it is. So it is what it does that counts-after all, that's what any primitive is introduced for in a theory in the first place (otherwise there would be little justification for having it). Thus, primitives are individuated by what they do, what their functional role in a theory is, and as a consequence two primitives that do the same job just turn out to be equivalent for all theoretical purposes and metaphysically equivalent as well: they just are one and the same thing referred to in two different ways. We have seen above that with respect to some traditional alleged points of disagreement, the views I have examined contain a primitive machinery that does the same job at the same place in the same way (that is, in a primitive way).

This is what I will call the "functional view" of primitives, it's the view that I have been using above and that I believe to be correct. But there is an alternative view, that I will call the "content view" of primitives, that claims that not only do primitives have a function in a theory but they also have a nature (a content). For instance, in the case of the Bundle Theory and the Substratum Theory this view insists that the unifying device involved in the former is a relation while the unifying device involved in the latter is a substratum and this just is not the same thing, however, similarly they may work.

Choosing between the functional view or the content view has important consequences for the sort of conclusions that can be drawn about the three pairs of relational and substantival theories. If the functional view is correct, the primitives used by the two sides of a debate just turn out to be the same thing and given the similarity of structure and equivalent explanatory power of the two allegedly competing theories, it appears that the difference between the relational side and the substantival side is no more than terminological. I leave it then an open question whether this terminological difference can be a good reason to prefer one side over the other. Hirsch $(2005,2007,2008)$, when making an equivalence claim about the debate between perdurantists and endurantists, insists that while there is only terminological difference between the two sides of the debate, the endurantist language is closer to ordinary language and so should be preferred. Alternatively, one can see closeness of theoretical terminology to ordinary language as irrelevant and simply claim that it does not matter at all which side of the debate one chooses to embrace-or better, that we should simply refrain from choosing. This question about which is the better terminology left aside, I would like to insist on the following: in all theories we have seen, and in metaphysical theories in general (we will see shortly some other examples below), the primitives do a big part of the job-indeed, without its primitives none of the views would even begin to work and primitives are used in all crucial places where a serious problem needs to be solved or an important phenomenon (like persistence, unity, ...) accounted for. Under the 
functional view, it is then no wonder that if the primitives turn out to be equivalent (since they are individuated by their theoretical role and the role is equivalent) then the theories that contain them appear to be no more than terminological variants. I will call this the "Strong Conclusion".

The content view of primitives allows for a Weak(er) Conclusion to hold. Indeed, even if it is accepted that the problem-solving primitives used by two sides of a debate are functionally equivalent, they still are, according to this view, different in nature and so the theories that contain them cannot in turn be said to be equivalentthat is, they cannot be said to be metaphysically equivalent, while of course they can be said to be theoretically equivalent given that they do the very same job in the same way. The Weak Conclusion then claims not that the two sides of the debate are merely terminological variants, since they postulate primitives that are metaphysically different, but that it is epistemically under-determined which one we should choose.

Let me now say why I think that the functional view is to be preferred. Take the case of the Bundle Theory and Substratum Theory: the defender of the content view will insist that one side's problem-solver is a relation and the other side's problem-solver is a substratum and so they are entities with a different nature. Now, I think there are two possibilities: either any difference that this can make will be a functional difference, or this is just stubbornly sticking to terminology. For instance, the defender of the content view might say that a substratum is 'ontologically independent', that is, it can exist without exemplifying any properties, while the relation of compresence cannot just 'be there' and relate nothing. But if that were to be a way to claim that there is a difference between the two primitives, then it would be a functional difference: there is something that one primitive can do (standing alone, not unifying anything) and that the other primitive cannot do. So this is not going to give the friend of the content view what she needs. But it could, of course, block any equivalence claim since this would actually show that the two unifying devices do not always play the same theoretical role in the same way, and are thus not equivalent-or at least the equivalence claim has to be restricted to some cases only, but cannot be generalized. This is of course something that I am open to: if it can be shown that the two unifying devices do not have exactly the same function, any of the (Weak or Strong) Conclusions could only be partial. Elsewhere ${ }^{7}$ I have discussed the case I just mentioned about 'independence' with the result that there actually is no difference with respect to the two primitives, but in principle it is an open possibility that a place where they do play a different role can be found (but until then, the equivalence claim holds). Anyway, even if this were the case, the difference between the two primitives would be a functional difference so such a case would count in favour of the functional view. In principle, we should always expect any difference between primitives to be functional, with no surprise: since primitives are introduced in the theory by the metaphysician who needs them because she cannot make the theory work without them, she'll typically always introduce primitives to do a theoretical job, otherwise she would not even bother

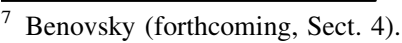


with them in the first place-this is why it seems to me quite obvious that any difference we could find between primitives will turn out to be functional. ${ }^{8}$ If someone insisted that on the top of the functions they play in the theory, primitives have a non-functional content, this would then amount to insisting that she postulates a difference that makes no difference-and I can only say that I see little reason for doing anything like that when building a metaphysical theory. Such an attitude towards primitives would be having an unreasonable soft spot for the words one uses-words like "substance", "substratum", "relation", and so on.

Perhaps the friend of the content view could argue that even if two primitives play the same theoretical role-that is, they have the same function-they still have a different nature and a non-functional way to see that is to embrace one of the two primitives as being more intuitive than the other. Something like this might have played an important role in the debate between substratum theorists and bundle theorists where the latter sometimes in this vein qualified the substratum as being a mysterious entity. So can intuitions help us to distinguish between two primitives that have the same function? I do not think so: there are some worries that arise here, showing that intuitions are not really pertinent in this matter. Firstly, intuitions do not seem to be relevant in the field of basic metaphysics that is just too abstract and theoretical for any useful intuitions to arise-unlike in other less basic debates; for instance, imaginary cases or Star Trek stories of duplication of persons in the debate about personal identity allow us to give rise to some carefully formulated and useful intuitions that can probably do some helpful work in the understating of our concept of a person and its conditions of persistence through time. But when fundamental and highly abstract metaphysical issues are concerned, such as those that we are concerned with in our case of the three pairs of relational and substantival ontologies, there just do not seem to be any useful intuitions around: these are not matters where anybody can have any intuitions, except, again, misleading intuitions that come from attachment to words like "substance" or "relation". Secondly, any intuitions suffer from being too unsettled and variable from one thinker to another and even over time for one and the same thinker, and even there conflicting intuitions, good and bad intuitions, as well as weak and strong intuitions can arise- thus, they do not seem to be a very reliable guide.

I think that this leaves us with the functional view, and with the consequence that if my arguments from Sects. 1 to 3 are correct, a Strong Conclusion can be drawn

\footnotetext{
${ }^{8}$ This is also the reason why I prefer to restrict my claim that the functional view is correct only to the case of primitives, and not all other cases of functionally equivalent non-primitive entities or bits of theories. For instance, you might think of the case of two properties that have each a certain function in a world $W$ and that 'switch' their function in a world $W^{\prime}$ while each keeping its identity: in such a case, it seems that something like the content view is to be preferred, with respect to the nature of properties. The difference between such cases and the case of primitives is that primitives are theoretical postulates that are introduced in a theory by a metaphysician who needs them to perform a certain job, a function, and so this is why I think there that the functional view holds, while properties are not just theoretical postulates and so it may very well be that with respect to properties the content view is more adequate since it seems at least prima facie more plausible that they have a nature that is not reducible only to their function. (But of course, the opposite may turn out to be true; all I want to say is that I wish here to restrict my claims only to the case of primitives.).
} 
about the three pairs of relational and substantival ontologies. ${ }^{9}$ (If you are not convinced by the functional view of primitives, then it is the Weak Conclusion that is in order-a Weak Conclusion that is still pretty strong.) To sum up some central points of my approach:

- theories such as the 6 theories discussed in this paper only work thanks to the job done by their primitives; this is of course to be expected and perfectly in order since otherwise there would be no reason to introduce the primitives in the first place

- thus, it is crucial to recognize that, indeed, these primitives are introduced by the theorist, they are theoretical postulates that are introduced to do a job: they are there for what they can do, they are functions within the theory

- as such, they are individuated by their functional role; this has the consequence that if two primitives have the same function they turn out to be not only theoretically but also metaphysically equivalent

- I have provided some examples as study cases of places where I think that allegedly competing theories exhibit such equivalent primitives and since these theories are also structurally extremely similar and since most of the work they do is done by their primitives, the Strong Conclusion, that is, a conclusion of metaphysical equivalence, can be drawn

I shall now conclude by examining two objections that can be made to my approach, both of which will allow me to refine the scope and the content of my conclusions. Put shortly, a first worry could be that I have considered here too substantival versions of the relational ontologies I used as examples. To take the case of relationism about time, one way to articulate this worry would be to claim that there may be, and there are, versions of relationism that do not appeal to a metaphysically loaded relation of simultaneity to get instants out of events, and that doing so is somehow for a relationist to betray the spirit of her own view, by mimicking her opponent's. The reason and the need for her to do so may then simply have come from the pressure of objections to relationism like the Shoemaker one-involved in a debate filled with considerations about global freezes and empty time, the relationist (at least the relationist I considered in this paper) then subtly has the tendency to adopt a substantival ideology and tools, disguised under relationist vocabulary. To my mind, if anything like this were true, it would be an interesting result in itself.

As a more general worry, one could also ask ${ }^{10}$ whether the method I recommend to use in order to see whether two theories are equivalent or not - that is, to look at how the theories and primitives work, how they function, to see whether any equivalence claim can be drawn-does not give us equivalence too easily and too cheaply. Indeed, in a very general way one could use my claims to say that

\footnotetext{
9 With the important proviso that I have here only partly and not fully examined the three debates, so any conclusion that can be drawn here is only partial. I have examined the three debates in more detail and with more completeness elsewhere (see Benovsky 2008); but most of all I hope that I have made plausible here the method to achieve the conclusion, that can be applied to other points of alleged disagreement between the two sides in the same way.

${ }^{10}$ I would like to thank Mark Heller for insisting on this.
} 
whenever two theories explain the same thing, they turn out to be equivalent. The materialist and the dualist both account, in their own terms, for the fact that I feel back pain this morning and for the having of qualia in general-does it then mean that I would say that they are equivalent, since they accomplish the same work in the end? Of course, this is not something that I want to say. Elsewhere, ${ }^{11}$ contra Hirsch (2008) and Miller (2005), I have for instance argued that following my method perdurantism and endurantism are not equivalent, they are only partly equivalent but there remain genuine substantial differences between these two sides of the persistence debate. Examining this debate here would be too long, so let me take another example that will allow me to say what is too cheap and what is not. Let us take three theories about the nature of ordinary material objects and see how they behave with respect to the traditional problem of "attribute agreement". The central questions these theories face are: (1) What are material objects (such as tables, computers, lizards, ...), what is their nature? (2) What are properties, what is their nature? (3) How do objects have their properties? It is better to consider views that answer all three questions, since if we took only one question at a time, we would never have theories 'complete enough' to be proper subjects of comparison and evaluation. A complete cartography of the various theories that provide answers to these questions would be a matter for a very long book, and would fall quite outside the scope and the purpose of this paper; but it is enough for my present concerns to use as an example a partial and quite schematic map, featuring what I take to be the three strongest, most representative, and typical views. ${ }^{12}$

Theory 1 Substrata that instantiate immanent universals

\begin{tabular}{|c|c|}
\hline Answer to Question 1 & $\begin{array}{l}\text { Objects are bare particulars (substrata) that instantiate immanent } \\
\text { (spatio-temporal, multiply locatable) universals }\end{array}$ \\
\hline \multirow[t]{2}{*}{ Answer to Questions 2 and 3} & $a$ is $F$ iff $a$ instantiates the universal $F$-ness \\
\hline & $\begin{array}{l}a \text { and } b \text { are both } F \text { ('share the same property') iff } a \text { and } b \text { both } \\
\text { instantiate the numerically same universal } F \text {-ness }\end{array}$ \\
\hline \multirow{3}{*}{$\begin{array}{l}\text { Primitives, and other } \\
\text { ontological commitments }\end{array}$} & Bare particulars (substrata) \\
\hline & Immanent universals \\
\hline & Non-relational instantiation \\
\hline \multirow[t]{2}{*}{ Main objections (and replies) } & $\begin{array}{l}\text { OBJ1: Infinite (Bradley-like) Regress, [REPLY: the primitive } \\
\text { instantiation is non-relational (Armstrong 1978)] }\end{array}$ \\
\hline & $\begin{array}{l}\text { OBJ 2: Immanent universals are unacceptable because multiple } \\
\text { location is absurd: "redness is at one meter from itself", [REPLY: } \\
\text { this intuition was made for objects, not for properties (Lewis 1983; } \\
\text { Loux 1998; Hawthorne and Cover 1998)] }\end{array}$ \\
\hline
\end{tabular}

\footnotetext{
11 Benovsky (2009).

12 If your preferred view is not listed here, just add it yourself; I am sure that you will get a table similar to the ones I have here. The references provided in these tables are not (always) references to the original places where the objection or point first appeared, rather they are references to places where it is, in my opinion, nicely articulated.
} 
Theory 1 continued

OBJ 3: Identity conditions of substrata. In virtue of what is a substratum distinct from another? No attributes or properties can distinguish between them. Loux (1998), [REPLY: They do have properties. For what it is to have properties, according to the substratum theory, is to instantiate universals (Sider 2006)]

OBJ 4: Substrata are unknowable and they cannot be experienced. Campbell (1981), [REPLY 1: let us concede that the introduction of bare substrata is incompatible with a rigorous empiricism, but [...] the constraints the empiricist imposes on the metaphysical enterprise are unreasonably stringent. Loux (1997, 1998), REPLY 2: Substrata do have properties, so the epistemological argument may be swiftly dispatched. We clearly can know what universals a thin particular instantiates, and so know what it is like; and in what other sense ought we be able to "know it"? (Sider 2006)]

OBJ 5: The idea of a bare entity with no essential attributes is incoherent, substrata have the property of not having any property (Loux 1997, 1998), [REPLY: only sparse properties count here, and 'having no property' is not sparse (Armstrong 1978; Sider 2006)]

Theory 2 Bundles of tropes

Answer to Question 1

Answer to Questions 2 and 3

Primitives, and other ontological commitments

Main objections (and replies)
Objects are bundles of compresent non-repeatable properties (tropes)

$a$ is $F$ iff a has among its members/constituents/parts an $F$-trope

$a$ and $b$ are both $F$ ('share the same property') iff $a$ and $b$ both have among their members/constituents/parts numerically different $F$ tropes that are exactly similar

Tropes (their existence and nature)

Compresence

Exact resemblance of tropes; resemblance

Possibilia + counterpart theory

OBJ1: variant of the problem with co-extensive properties (Manley 2002), (REPLY: use possibilia)

OBJ2: if F-ness is the set of all resembling tropes, since sets have their members necessarily, there could not be a single object that is $F$ in addition to those that are there (Loux 1998), (REPLY: use possibilia)

OBJ3: the problem of naturalness (imperfect community difficulty), [REPLY: does not arise if we use exact resemblance (a primitive); more in Manley (2002)]

OBJ 4: Infinite Regress-if we have exactly resembling tropes then the resemblance relations must resemble, and these must resemble, and so on. [REPLY: there is no regress thanks to the primitive fact that $a$ and $b$ resemble each other. We do not have two tropes and a relation of resemblance, but two tropes, and given these two, they resemble (Simons 1994)]

OBJ 5: Problem with change. If an individual is identified with a bundle of properties, then if one of the properties changes, the bundle is not the same, and so, the individual who is the bundle is not the same. (Van Cleve 1985), [REPLY: use four-dimensionalism (Hawthorne and Cover 1998), or also endurantism (Benovsky 2008)]

OBJ 6: Modal analogue of the problem of change above. (REPLY: use modal counterpart theory)

OBJ 7: Properties are not the kind of entities that are capable of independent existence, they are not suitable to be ultimate constituents of reality (Armstrong 1997). [REPLY: this is no more than an undefended incredulous stare (Hawthorne and Cover (1998)] 
Theory 3 Resemblance Nominalism, without paradigms

\begin{tabular}{|c|c|}
\hline Answer to Question 1 & Objects are not analyzed by this theory, they are taken as primitive \\
\hline \multirow[t]{2}{*}{ Answer to Questions 2 and 3} & $\begin{array}{l}a \text { is } F \text { iff a resembles all the } F \text { s (the right-hand side being the more } \\
\text { fundamental) }\end{array}$ \\
\hline & $\begin{array}{l}a \text { and } b \text { are both } F \text { ('share the same property') iff they are both } \\
\text { members of the same resemblance class }\end{array}$ \\
\hline \multirow{3}{*}{$\begin{array}{l}\text { Primitives, and other } \\
\text { ontological commitments }\end{array}$} & Objects (answer to Question 1) \\
\hline & $\begin{array}{l}\text { The fact that } a \text { and } b \text { resemble each other (but there is not a relation of } \\
\text { resemblance between } a \text { and } b \text {; resemblance is a relational fact without } \\
\text { there being such a relation) }\end{array}$ \\
\hline & Possibilia + counterpart theory \\
\hline \multirow[t]{6}{*}{ Main objections (and replies) } & $\begin{array}{l}\text { OBJ1: the possibility that there is only one object that is } F \text { (Armstrong } \\
\text { 1978), [REPLY: use possibilia (Rodriguez-Pereyra 2002)] }\end{array}$ \\
\hline & $\begin{array}{l}\text { OBJ2: co-extensive properties, and the companionship difficulty } \\
\text { (Armstrong 1978; Campbell 1981; Jackson 1997; Manley 2002), } \\
\text { [REPLY: use possibilia (Lewis 1986; Rodriguez-Pereyra 2002)] }\end{array}$ \\
\hline & $\begin{array}{l}\text { OBJ3: the problem of naturalness (the imperfect community difficulty) } \\
\text { (Armstrong 1978; Manley 2002), (REPLY: (Rodriguez-Pereyra } \\
\text { 2002) using primitive resemblance) }\end{array}$ \\
\hline & $\begin{array}{l}\text { OBJ5: Russell's regress (Russell 1912; Armstrong 1978), (REPLY: } \\
\text { there is no regress (Rodriguez-Pereyra } 2002 \text { using the primitive fact } \\
\text { that } a \text { and } b \text { resemble each other) }\end{array}$ \\
\hline & $\begin{array}{l}\text { OBJ6: resemblance nominalism and causality; causal powers of } \\
\text { objects should depend on how objects are and not on how they are } \\
\text { related to other objects (Armstrong 1978), (REPLY: causal powers do } \\
\text { depend on how objects are, while objects are the way they are in } \\
\text { virtue of resembling other objects-this is among the primitives of } \\
\text { resemblance nominalism (G. Rodriguez-Pereyra, private } \\
\text { communication) }\end{array}$ \\
\hline & $\begin{array}{l}\text { OBJ7: } a \text { resembles } b \text { because } a \text { is } F \text { and } b \text { is } F \text {, not the other way } \\
\text { around (Armstrong 1978), (REPLY: this shows that on this point } \\
\text { resemblance nominalism goes against intuition, but the view has } \\
\text { theoretical virtues that outweigh this (Rodriguez-Pereyra 2002) }\end{array}$ \\
\hline
\end{tabular}

I do not claim that these tables are complete and exhaust all the (variants of) objections there are against the three theories. But I do claim that all main objections are there, and that these tables are complete enough to allow me to suggest that none of the objections listed above is decisive, in the sense that none of the three theories can be said to be clearly eliminated by it, and neither is one of the three theories clearly eliminated by the collective force of the objections to it. But the main point that I want to emphasize here is that, as for all of the six theories we have seen above, the primitives of each theory do a big part of the job, when it comes to answering the initial question(s) and replying to objections. It is only if we grant the views their primitives (and ontological commitments) that they can face all the objections. I am not saying, of course, that the three theories have all their objections easily answered and that they are problem-free, all I am saying is that all three views can plausibly face the objections that arise against them, with the crucial help of their primitives. 
When one considers these primitives, one encounters problem-solvers exactly as in the case of the six relational and substantival ontologies we have seen above. Furthermore, some of these problem-solvers seem to play the same crucial role in the same crucial place: one can ask "In virtue of what are two exactly resembling tropes exactly similar?"; another can ask "In virtue of what are these two instances of $F$-ness instances of $F$-ness?"; another can ask "In virtue of what do these two particulars resemble each other?". Of course, these are unfair questions since they ask the opponent to go beyond her primitives. But they illustrate the point I want to emphasize-all three views answer the question of attribute agreement by using their primitive machinery: the relation of exact resemblance between tropes, the instantiation of the same universal, or the fact that $a$ and $b$ resemble each other. At the same crucial places, all three views introduce a primitive with the same function: primitively answer the question ("In virtue of what $x$ and $y$ are both $F$ ?"). If one agrees to be fair to one's opponents, granting them the same right to introduce a primitive as one grants herself, it seems to me hard to claim that any of the primitive ways of answering the question is better than the others. So, am I saying that these three theories are equivalent, since they "do the same job" (that is, account for attribute agreement) by appealing to primitive problem-solvers? No, for two inter-related reasons. The three theories are structurally quite different so even if it were true that they all employ problem-solvers to answer one of their central challenges, they still do not use their problem-solvers in the same way (i.e. at the same place in the theory). For instance, Theory 3 contains only objects and the problem-solver (primitive resemblance), while Theory 1 contains bare objects and universals and the problem-solver (primitive instantiation), so the two views are structurally distinct. Thus, such views are not equivalent since while they do the same job at a very general level (that is, they all account for attribute agreement) they do not do their job in the same way, even if we were ready to accept that their problem-solvers are equivalent. In order to claim that two theories are equivalent, not only must they do the same overall job, but they must also do it in the same way which in turn means that they must have a structure similar enough to allow for this (in addition to the fact that they employ the same problem-solvers). Whether an equivalence claim is too cheap or not depends then on the level of detail the claim provides. If such a claim is very general, it is very cheap; but if it is elaborated in detail and shows how the inner workings of two theories are similar, then it is worth your money (Theory 2).

What this shows, en passant, is that in order to get any meta-metaphysical results, such as equivalence claims between two theories, we need first to do first-level metaphysics and study how things work at a great level of detail, to get any worthwhile results. Thus, any meta-metaphysical claims should not be made in a too general way but should always be supported by low-level considerations. ${ }^{13}$

\footnotetext{
13 For very helpful comments and discussions, I would like to thank Fabrice Correia, Akiko Frischhut, Lynda Gaudemard, Richard Glauser, Ghislain Guigon, Mark Heller, Max Kistler, Jessica Leech, Dan López de Sa, Jonathan Lowe, Anne Meylan, Kevin Mulligan, Laurie Paul, Jonathan Schaffer, Gianfranco Soldati, and John Zeimbekis. Special thanks go to two anonymous referees of Erkenntnis whose valuable comments helped me to improve the final version of this paper.
} 


\section{References}

Armstrong, D. M. (1978). Nominalism and realism. Cambridge: Cambridge University Press.

Bennett, K. (2008). Composition, colocation, and metaontology. In D. Chalmers, D. Manley, \& R. Wasserman (Eds.), Metametaphysics. Oxford: Oxford University Press.

Benovsky, J. (2008). The bundle theory and the substratum theory: Deadly enemies or twin brothers? Philosophical Studies, 141, 175-190.

Benovsky, J. (2009). Eternalist theories of persistence through time: Where the differences really lie. Axiomathes, 19(1).

Benovsky, J. (forthcoming). The relationist and substantivalist theories of time: Foes or friends? European Journal of Philosophy.

Chalmers, D. (2008). Ontological anti-realism. In D. Chalmers, D. Manley, \& R. Wasserman (Eds.), Metametaphysics. Oxford: Oxford University Press.

Ehring, D. (2001). Temporal parts and bundle theory. Philosophical Studies, 104, 163-168.

Forbes, G. (1993). Time, events, and modality. In R. Le Poidevin \& M. MacBeath (Eds.), The philosophy of time. Oxford: Oxford University Press.

Hawthorne, J., \& Cover, J. (1998). A world of universals. Philosophical Studies, 91, 205-219.

Hirsch, E. (2005). Physical-object ontology, verbal disputes, and common sense. Philosophy and Phenomenological Research, 70, 67-97.

Hirsch, E. (2007). Ontological arguments: Interpretive charity and quantifier variance. In J. Hawthorne, T. Sider, \& D. Zimmerman (Eds.), Contemporary debates in metaphysics. Oxford: Blackwell.

Hirsch, E. (2008). Ontology and alternative languages. In D. Chalmers, D. Manley, \& R. Wasserman (Eds.), Metametaphysics. Oxford: Oxford University Press.

Lewis, D. (1983). New work for a theory of universals. Australasian Journal of Philosophy 61.

Lewis, D. (1986). On the plurality of worlds. Oxford: Blackwell.

Loux, M. J. (1997). Beyond substrata and bundles. Reprinted in Macdonald and Laurence 1998. Contemporary readings in the foundations of metaphysics. Oxford: Basil Blackwell.

Loux, M. J. (Ed.). (1998). Metaphysics: A contemporary introduction. London: Routledge.

Manley, D. (2002). Properties and resemblance classes. Noûs, 36, 75-96.

Miller, K. (2005). The metaphysical equivalence of three and four dimensionalism. Erkenntnis, 62, 91-117.

Paul, L. A. (forthcoming). Identity, indiscernibility and objects. In Loux, Paul and van Inwagen, Oxford: Oxford University Press.

Rodriguez-Pereyra, G. (2002). Resemblance nominalism, a solution to the problem of universals. Oxford. Russell, B. (1912). Problems of philosophy. Oxford: Oxford University Press.

Shoemaker, S. (1969). Time without change. Journal of Philosophy, 66, 363-381.

Shoemaker, S. (1997). Self and substance. Philosophical Perspectives, 11.

Sider, T. (2001). Criteria of personal identity and the limits of conceptual analysis. Philosophical Perspectives, 15, 189-209.

Sider, T. (2006). Bare particulars. Philosophical Perspectives, 20, 387-397.

Sider, T. (2007). Which disputes are substantive. Unpublished.

Sider, T. (2008). Ontological realism. In D. Chalmers, D. Manley, \& R. Wasserman (Eds.), Metametaphysics. Oxford: Oxford University Press.

Simons, P. (1994). Particulars in particular clothing: Three trope theories of substance. Philosophy and Phenomenological Research, 54, 553-575.

Van Cleve, J. (1985). Three versions of the bundle theory. Reprinted in Loux 2001.

Yablo, S. (2008). Must existence questions have answers. In D. Chalmers, D. Manley, \& R. Wasserman (Eds.), Metametaphysics. Oxford: Oxford University Press. 